\title{
THE OUTCOME OF CARDIAC CASES IDENTIFIED PERI-NATALLY IN A DISTRICT GENERAL HOSPITAL IN UNITED KINGDOM
}

\author{
P. Siddhi, K. Veni, R. Saravanan, A. Tillet, V. Karruppaswamy \\ Colchester General Hospital, Colchester, UK
}

\begin{abstract}
Aims: To identify the annual incidence of congenital heart disease (CHD) in our population and to assess the pick up rate during antenatal ultrasound.
\end{abstract}

Methods: Retrospective analysis of cases diagnosed with CHD between January 2007 - April 2010.

Results: Forty-four cases of CHD were diagnosed during the study period. Of these, 19 cases were diagnosed antenatally and 25 cases postnatally.

Of the 19 cases:

- 7 four-chamber anomalies

- 7 out-flow tract anomalies and

- 5 cases of cardiomegaly

5 cases were terminated [associated with syndromes], 2 resulted in miscarriages, 5 suffered neonatal deaths and 7 survived (survival rate- $37 \%$ ).

Of the 25 cases diagnosed postnatally:

Twenty babies were term deliveries and 5 were pre-term. 16 -cases $(64 \%)$ were diagnosed within the $1^{\text {st }}$ month of delivery.

Eight (32\%) of the 25 cases were cyanotic CHD.

Following are the specific diagnosis of postnatal CHD.

- Transposition of great arteries- 4

- Aortic stenosis- 1

- Pulmonary stenosis- 5

- Septal defects- 7

- Ebstein anomaly- 1

- Tetralogy of Fallot- 3

- Coarctation of aorta- 2

- Dextrocardia- 1

- Coronary artery fistula- 1

16-cases were treated with surgical corrections and 9-cases treated conservatively. 24-neonates survived (survival rate- 96\%).

Conclusion: Annual incidence rate of CHD in our population was $1.4 \%$ with $43 \%$ of cases identified during antenatal scans. Following the audit, following measures have been introduced.

- Merging antenatal cardiac clinics and paediatric cardiac clinics

- “Tiny Tickers"- The Regional Foetal Cardiac Training Group for the Ultrasonographers are introducing a training program. 